\title{
Type 2 diabetes and cancer: umbrella review of meta-analyses of observational studies
}

\author{
@) (1) $\Theta$ OPEN ACCESS
}

\author{
Konstantinos K Tsilidis assistant professor ${ }^{12}$, John C Kasimis PhD student ${ }^{1}$, David S Lopez assistant \\ professor $^{3}$, Evangelia E Ntzani assistant professor ${ }^{1}$, John P A loannidis professor ${ }^{4}$
}

${ }^{1}$ Department of Hygiene and Epidemiology, University of loannina School of Medicine, loannina, 45110, Greece; ${ }^{2}$ Department of Epidemiology and Biostatistics, School of Public Health, Imperial College London, London, UK; ${ }^{3}$ Division of Epidemiology, University of Texas School of Public Health, 1200 Pressler Street, Houston, TX 77030, USA ${ }^{4}$ Stanford Prevention Research Center, Department of Medicine, and Department of Health Research and Policy, Stanford University School of Medicine, and Department of Statistics, Stanford University School of Humanities and Sciences, 1265 Welch Rd., Stanford, CA, 94305, USA

\begin{abstract}
Objectives To summarise the evidence and evaluate the validity of the associations between type 2 diabetes and the risk of developing or dying from cancer.
\end{abstract}

Design An umbrella review of the evidence across meta-analyses of observational studies of type 2 diabetes with risk of developing or dying from any cancer.

Data sources PubMed, Embase, Cochrane database of systematic reviews, and manual screening of references.

Eligibility criteria Meta-analyses or systematic reviews of observational studies in humans that examined the association between type 2 diabetes and risk of developing or dying from cancer.

Results Eligible meta-analyses assessed associations between type 2 diabetes and risk of developing cancer in 20 sites and mortality for seven cancer sites. The summary random effects estimates were significant at $\mathrm{P}=0.05$ in 20 meta-analyses (74\%); and all reported increased risks of developing cancer for participants with versus without diabetes. Of the 27 meta-analyses, eventually only seven (26\%) compiled evidence on more than 1000 cases, had significant summary associations at $\mathrm{P} \leq 0.001$ for both random and fixed effects calculations, and had neither evidence of small study effects nor evidence for excess significance. Of those, only six (22\%) did not have substantial heterogeneity $\left(I^{2}>75 \%\right)$, pertaining to associations between type 2 diabetes and risk of developing breast, cholangiocarcinoma (both intrahepatic and extrahepatic), colorectal, endometrial, and gallbladder cancer. The 95\% prediction intervals excluded the null value for four of these associations (breast, intrahepatic cholangiocarcinoma, colorectal, and endometrial cancer).

Conclusions Though type 2 diabetes has been extensively studied in relation to risk of developing cancer and cancer mortality and strong claims of significance exist for most of the studied associations, only a minority of these associations have robust supporting evidence without hints of bias.

\section{Introduction}

Almost 400 million people throughout the world have diabetes, $85-95 \%$ of whom have type 2 disease. ${ }^{1}$ Cancer is the second leading cause of death in economically developed countries and the third leading cause of death in developing countries. ${ }^{2}$ Multiple studies and meta-analyses have claimed that type 2 diabetes is associated with an increased risk of cancer at several sites including liver, pancreas, endometrium, colorectum, breast, and bladder, and a decreased risk of prostate cancer. ${ }^{3}$ The observed associations between type 2 diabetes and cancer could be either causal (for example, caused by hyperglycaemia or hyperinsulinaemia) or because of confounding from common risk factors such as adiposity. If causal, these associations might be of great importance for public health given the substantial global burden of these diseases. ${ }^{2}$ It is also possible, however, that some claimed associations could be caused by biases in the literature, in particular selective reporting biases favouring the publication of significant associations ${ }^{4}$ and causing either false positives ${ }^{5}$ or inflated estimates of association. ${ }^{6}$ Biases have been suspected, documented, or debated in observational epidemiology of cancer for multiple putative carcinogens, risk factors, biomarkers, and prognostic factors. ${ }^{7-13}$

To understand the strength of evidence and extent of potential biases in the claimed associations between type 2 diabetes and risk of developing cancer, we performed an umbrella review of the evidence across published meta-analyses or systematic 
reviews. We summarised the evidence on the incidence of cancer and cancer mortality for each site that has been studied in its association with type 2 diabetes; described the magnitude, direction, and significance of the observed associations; evaluated whether there are hints of biases in this evidence and how they manifest; and identified which are the most robust associations without potential biases.

\section{Methods}

\section{Literature search}

Two researchers (JCK and KKT) independently searched PubMed, Embase, and the Cochrane database of systematic reviews from inception to the end of 2013 for meta-analyses or systematic reviews of epidemiological studies investigating the association between type 2 diabetes and risk of any developing cancer or death from cancer using the following search algorithm: "(diabetes) AND (cancer OR carcinoma OR neoplasia OR tumor OR neoplasm OR maligna*) AND (meta-analysis OR systematic review)". A manual review of references from eligible systematic or narrative reviews was also performed. The titles, abstracts, and full texts of the resulting papers were examined in detail, and discrepancies were resolved by consensus.

\section{Eligibility criteria and data extraction}

Articles were eligible if the authors had performed a systematic search to identify pertinent studies. We included only meta-analyses or systematic reviews of epidemiological studies in humans. Randomised controlled trials were unavailable for our research question. We excluded studies in which type 2 diabetes was not the exposure of interest (such as type 1 and gestational diabetes) and cancer incidence or mortality was not the outcome of interest and meta-analyses of prognostic studies associating type 2 diabetes and mortality among patients with cancer. Meta-analyses or systematic reviews that did not present study specific data (relative risks, $95 \%$ confidence intervals, and numbers of cases/population) were also excluded. If an article presented separate meta-analyses on more than one eligible outcome (such as cancer incidence, mortality for one or more cancer sites), those were assessed separately. Whenever more than one meta-analysis existed on the same scientific question, the meta-analysis with the largest number of studies was selected, but we conducted sensitivity analyses to assess the concordance of the summary associations (direction, magnitude, and significance) in these duplicate meta-analyses. From each eligible meta-analysis, two investigators (JCK and KKT) abstracted information independently on first author, year of publication, outcome examined, number of included studies, and reported summary meta-analytic estimates using both fixed and random effects methods. For each of the included studies in each eligible meta-analysis, we recorded the epidemiological design (case-control or cohort), the number of cases and controls (in case-control studies) or the number of cases and population participants (in cohort studies), and the maximally adjusted relative risk (reported as odds ratio for case-control studies and hazard ratio or standardised incidence/mortality ratio for cohort studies) and 95\% confidence intervals. Most eligible

meta-analyses or systematic reviews did not assess the quality of the included studies, and this task was beyond the scope of this umbrella review, but we did assess essential features of the quality of the evidence at the meta-analysis level.

\section{Assessment of summary effects and heterogeneity}

We estimated the summary effects using both fixed and inverse variance random effects methods. ${ }^{14}$ When the original meta-analyses presented study results separately by sex, we combined the summary effect per study first using random effects methods before conducting the overall meta-analysis. Methods of fixed effect meta-analysis are based on the assumption that a single common (or "fixed") effect underlies every study in the meta-analysis without any heterogeneity between studies. A random effects synthesis makes the assumption that individual studies are estimating different effects, which are assumed to have a distribution. The random effects meta-analysis is performed to estimate the mean of this distribution of effects across different studies and the uncertainty about that mean (95\% confidence interval). We also calculated the $95 \%$ prediction intervals for the summary random effects estimates, which further account for heterogeneity between studies and indicate the uncertainty for the effect that would be expected in a new study examining that same association. ${ }^{15}$ The 95\% prediction interval shows where the true effects are for $95 \%$ of the studies from the population of studies that are synthesised or similar (exchangeable) studies that might be done in the future.

We assessed heterogeneity between studies using the $\mathrm{P}$ value of the $\chi^{2}$ based Cochran $\mathrm{Q}$ test and the $\mathrm{I}^{2}$ metric of inconsistency; this could reflect either genuine diversity or bias. The Q test is obtained by the weighted sum of the squared differences of the observed effect in each study minus the fixed summary effect. ${ }^{16}$ The $\mathrm{I}^{2}$ metric ranges between $0 \%$ and $100 \%$ and is the ratio of variance between studies over the sum of the variances within and between studies; its $95 \%$ confidence intervals were calculated according to the method of Ioannidis and colleagues. ${ }^{17}$

\section{Assessment of small study effects}

We examined whether there is an indication for small study effects - that is, if small studies tend to give higher risk estimates than large studies. Small study effects can indicate publication and other reporting biases, but they can also reflect genuine heterogeneity, chance, or other reasons for differences between small and large studies. ${ }^{18} \mathrm{We}$ used the regression asymmetry test proposed by Egger for this assessment. ${ }^{19}$ Alternative tests proposed by Harbord or Peters have better properties when applied to dichotomous outcomes compared with the Egger test, but their calculation requires data from $2 \times 2$ tables. ${ }^{20}{ }^{21}$ The $2 \times 2$ table data, however, were not reported by individual studies in the included meta-analyses, and most meta-analyses used confounder adjusted estimates of effect in single studies rather than unadjusted relative risks calculated from $2 \times 2$ tables. We also calculated the association between type II diabetes and risk of developing cancer after extrapolating the Egger regression line on a funnel plot to a theoretical study of infinite sample size (with a standard error of zero), which has been proposed to produce a meta-analytical estimate that can be regarded as free from small study bias. ${ }^{22} 23$

\section{Evaluation of excess significance}

We applied the excess significance test to investigate whether the observed number of studies $(\mathrm{O})$ with nominally significant results ("positive" studies, $\mathrm{P}<0.05$ ) was different from the expected number of significant results $(\mathrm{E}) .^{24}$ The expected number of significant studies is calculated in each meta-analysis from the sum of the statistical power estimates for each component study. The estimated power of each component 
study depends on the plausible effect size for the tested association between type 2 diabetes and cancer. The actual size of the true effect in each meta-analysis is not known, but it can be assumed to be the effect of the largest study in each meta-analysis, as previously described.$^{25}$ The largest study was defined based on the smallest standard error. Sensitivity analyses were conducted based on the largest number of cancer cases or the largest total number of participants, but the results did not differ (not shown). The statistical power of each study was calculated with an algorithm from a non-central $t$ distribution, as previously shown. ${ }^{926}$ When we repeated the power calculations in a subset of our sample using logistic regression or Cox proportional hazards models, ${ }^{27}{ }^{28}$ we observed similar estimates. Excess significance for single meta-analyses was claimed at $\mathrm{P}<0.10$ (one sided $\mathrm{P}<0.05$ with $\mathrm{O}>\mathrm{E}$ as previously proposed). ${ }^{24}$

\section{Associations meeting further criteria}

We further identified associations for which the summary fixed and random effects estimates showed strong evidence of significance $(\mathrm{P}<0.001$; a threshold that has been suggested to substantially reduce the number of false positive findings ${ }^{29-31}$ ); were based on evidence from more than 1000 cases; did not have large heterogeneity between studies $\left(\mathrm{I}^{2}<75 \%\right)$; their $95 \%$ prediction interval excluded the null value; extrapolation of the Egger regression line for infinite sample size still suggested a nominally significant effect (95\% confidence interval excluding the null value); and had no evidence for excess significance. All calculations were performed in STATA version 12 .

\section{Results}

\section{Description of meta-analyses}

Of the 9667 articles initially identified in PubMed, Embase, and Cochrane databases, we finally selected 18 meta-analyses (all systematic reviews conducted a meta-analysis) including 27 comparisons (fig $1 \Downarrow$ ). Reviews included associations of type 2 diabetes with risk of diagnosis $(n=20)$ or death $(n=7)$ from bladder, ${ }^{32}$ breast ${ }^{33}$ cholangiocarcinoma (intrahepatic and extrahepatic),${ }^{34}$ colorectal,${ }^{35}$ endometrial,${ }^{36}$ oesophageal,${ }^{37}$ gallbladder, ${ }^{38}$ gastric,${ }^{39}{ }^{40}$ hepatocellular, ${ }^{41}$ kidney, ${ }^{42}$ leukaemia,${ }^{43}$ lung ${ }^{44}$ multiple myeloma,${ }^{43}$ non-Hodgkin's lymphoma, ${ }^{43}$ ovarian, ${ }^{45}$ pancreatic, ${ }^{46}$ prostate,${ }^{47}$ thyroid,${ }^{48}$ and total cancer. ${ }^{49}$ Table $1 \Downarrow$ summarises these 27 independent meta-analyses that included 474 individual study estimates. There were five to 45 study estimates combined per meta-analysis with a median of 14 studies. The median number of cases and population/controls in each meta-analysis was 7651 and 2556574 , respectively. The lowest number of cases in a meta-analysis was 103 , but all except for two meta-analyses (endometrial and hepatocellular cancer mortality) included more than 1000 cases. Of the 474 individual studies included in this umbrella review, $119(25 \%)$ were case-control studies and $355(75 \%)$ were cohort studies, 59 of which were retrospective cohorts or record linkage studies that used an external comparison group and thus calculated standardised incidence or mortality ratios. All 474 studies provided adjusted risk estimates, but 135 (28\%) studies adjusted for only one or two variables (usually age and sex) as reported in the original meta-analyses. We did not encounter any studies that could be affected by potential overadjustment after controlling for glucose, insulin, or glycosylated haemoglobin. We found more than one published meta-analysis for 13 cancer outcomes: incidence from bladder ( $\mathrm{n}=5$ meta-analyses), breast $(n=3)$, colorectal $(n=5)$, endometrial $(n=2)$, gastric $(n=2)$, hepatocellular $(n=3)$, non-Hodgkin's lymphoma $(n=3)$, pancreatic $(n=2)$, and prostate $(n=4)$ cancers; and mortality from breast $(n=2)$, colorectal $(n=3)$, endometrial $(n=2)$, and hepatocellular $(n=2)$ cancers. For most of these duplicate meta-analyses, there was agreement in principle on the direction, magnitude, and significance of the summary associations (table $\mathrm{A}$ in appendix). We observed small differences for the summary associations of type 2 diabetes and risk of mortality from hepatocellular cancer and incidence of prostate cancer, but the assessment of bias and of other features for the overall quality of the evidence were identical in these meta-analyses (table A in appendix).

\section{Summary effect size}

Out of the 27 meta-analyses (table $1 \Downarrow$ ), the summary random effects estimates were significant at $\mathrm{P} \leq 0.05$ in 20 meta-analyses (74\%), whereas the summary fixed effects were significant in 25 meta-analyses $(93 \%)$. When we used $\mathrm{P} \leq 0.001$ as a threshold for significance, 16 (breast, colorectal, endometrial, gastric, kidney and total cancer mortality, and gastric, kidney, lung, leukaemia, multiple myeloma, non-Hodgkin's lymphoma, ovarian, prostate, thyroid and total cancer incidence) and five (endometrial and kidney cancer mortality, and multiple myeloma, ovarian and thyroid cancer incidence) meta-analyses did not produce significant summary results using the random and fixed effects methods, respectively. All 11 associations with $\mathrm{P} \leq 0.001$ by random effects suggested increased risks of cancer. Table 1 also shows the effect of the largest study included in each meta-analysis. $\Downarrow$ Most $(n=21,78 \%)$ of the largest study effects in each meta-analysis were nominally significant at $\mathrm{P} \leq 0.05$, of which 19 showed increased risks. The effects of the largest studies were more conservative than the summary effects of the meta-analysis in $11(41 \%)$ of the 27 meta-analyses. When we calculated $95 \%$ prediction intervals, however, in only four associations was the null value excluded (for incidence of breast cancer, intrahepatic cholangiocarcinoma, colorectal cancer, and endometrial cancer).

\section{Heterogeneity between studies}

The $\mathrm{Q}$ test showed significant heterogeneity $(\mathrm{P} \leq 0.10)$ for 24 (89\%) meta-analyses (table $2 \Downarrow$ ). There was moderate to high heterogeneity $\left(\mathrm{I}^{2}=50-75 \%\right)$ in four meta-analyses and high heterogeneity $\left(\mathrm{I}^{2}>75 \%\right)$ in 16 (incidence of bladder, gastric, hepatocellular, kidney, lung, leukaemia, multiple myeloma, non-Hodgkin's lymphoma, pancreatic, and prostate cancer; and mortality from breast, colorectal, gastric, hepatocellular, and total cancer). Uncertainty around the heterogeneity estimates was often large, as reflected by wide $95 \%$ confidence intervals of the $\mathrm{I}^{2}$. When we calculated summary results separately in case-control and cohort (separately for prospective and retrospective cohorts) studies in a post hoc attempt to account for this large heterogeneity, we found no strong trend for the summary estimates in prospective cohort studies to be more conservative than the estimates in case-control studies, and the heterogeneity remained large for all study designs (tables B-D in appendix). None of the original meta-analyses reported significantly different summary estimates for type 2 diabetes and cancer by sex (data not shown).

\section{Small study effects}

There was no evidence for the presence of small study effects according to Egger's test (table 2) $\Downarrow$, except for incidence of endometrial and hepatocellular cancer, where $\mathrm{P}<0.10$ with more conservative effects in the larger studies. We calculated nominally significant summary estimates for 17 associations 
(63\%) after extrapolating the Egger regression line on a funnel plot to an infinitively large study (table 2 ).

\section{Excess significance}

Five meta-analyses (on incidence of bladder, hepatocellular, and kidney cancer and multiple myeloma and non-Hodgkin's lymphoma) had evidence of a significant excess of "positive" studies when the plausible effect was assumed to be equal to the effect of the largest study (table $2 \Downarrow$ ). Across all 474 studies, there was no overall evidence of excess significance, as 213 individual studies observed nominally significant associations but the expected number of significant studies was 230 .

\section{Associations meeting further criteria}

Of the 27 meta-analyses, eventually only seven (26\%) compiled evidence on more than 1000 cases, had significant summary associations at $\mathrm{P} \leq 0.001$ for both random and fixed effects calculations, and had neither evidence of small study effects (as manifested in a nominally significant association for an infinitively large study) nor evidence for excess significance (fig $2 \Downarrow$, tables 1 and $2 \Downarrow \Downarrow$ ). All these seven meta-analyses pertained to associations between type 2 diabetes and cancer incidence outcomes (breast, cholangiocarcinoma (both intrahepatic and extrahepatic), colorectal, endometrial, gallbladder, and pancreas). Of those, only six (22\%) meta-analyses did also not have substantial heterogeneity $\left(\mathrm{I}^{2}>75 \%\right.$ ), pertaining to associations with developing of breast, cholangiocarcinoma (both intrahepatic and extrahepatic), colorectal, endometrial and gallbladder cancer. The $95 \%$ prediction intervals excluded the null value for four of these associations, as shown in figure $2 \Downarrow$ (for incidence of breast, intrahepatic cholangiocarcinoma, colorectal and endometrial cancer). We also conducted a sensitivity analysis noting how many associations would satisfy the same criteria but with $\mathrm{P} \leq 0.05$ for the summary random and fixed effects calculations, but we eventually arrived at the same four associations.

\section{Discussion}

\section{Principal findings and possible explanations}

Our umbrella review examined the current evidence from meta-analyses on the link between type 2 diabetes and cancer for 20 different cancer sites. Most summary meta-analytic estimates yielded nominally significant results, with the risk for most cancers being higher in people with rather than without diabetes, except for prostate cancer, where the risk was lower in individuals with diabetes. Only a minority of these associations, however, had strongly significant results with no suggestion of bias, as can be inferred by substantial heterogeneity between studies, small study effects, and excess significance. The most robust evidence was seen for breast cancer, intrahepatic cholangiocarcinoma, colorectal cancer, and endometrial cancer, where the $95 \%$ prediction intervals also excluded the null value.

A possible association between type 2 diabetes and risk of developing cancer has long been speculated, as these diseases are diagnosed within the same individual more commonly than would be expected by chance. ${ }^{3}$ Most of the meta-analyses (20/27) that we critically appraised also reported a nominally significant result, but most of them had substantial heterogeneity that could not be readily explained by the epidemiological design, sex, or other important determinants for risk of developing cancer as reported here and in the original meta-analyses. When we took this substantial heterogeneity into account when calculating $95 \%$ prediction intervals (which provide a range for the association in a potential new original study), only the associations between type 2 diabetes and risk of developing breast cancer, intrahepatic cholangiocarcinoma, colorectal cancer, and endometrial cancer remained nominally significant. This result does not necessarily mean that only these four associations are genuine. It does, however, suggest that there is still substantial uncertainty about the other nominally significant associations. The large observed heterogeneity and some hints of bias in several of these meta-analyses suggest that some of these observed associations could be false positives or inflated. ${ }^{5}$

Empirical evidence suggests that biased observational associations might be common in the literature on cancer epidemiology. ${ }^{7-13}$ Usual suspected mechanisms that can operate are selective non-publication of "negative" results and selective choice of reporting analyses and outcomes that have stronger effects. ${ }^{51}$ The extent to which the literature on diabetes and cancer is affected by such biases is difficult to prove definitively. That only five to 45 published studies are available for each of these associations, however, is concerning. Diabetes and cancer are routine diagnoses, and information on their occurrence has probably been collected in a large number of observational datasets, perhaps in the range of hundreds. If one were to also include hospital records, there are probably thousands of datasets that could generate estimates of associations between type 2 diabetes and incidence and mortality for each cancer. Only a fraction of them are probably assessed, a smaller fraction is written up for publication, and an even smaller fraction is published, after peer review that is filtered by reviewers who might have specific beliefs about the presence of these associations. It is possible that only a small proportion of the potentially available data are eventually included in published analyses.$^{51}$ Ideally, one hopes that peer review has selected the best studies and the best data for publication. It is also possible, however, that bias in this literature could manifest itself as confirmation/allegiance bias. ${ }^{52}{ }^{53}$ If there is strong belief in the specialty about the association between type 2 diabetes and risk of developing cancer, research efforts are under pressure to conform to this hypothesis when and if they are to be published.

Some associations warrant further discussion. The associations between type 2 diabetes and risk of developing hepatocellular and pancreatic cancer did not pass our evaluation for robust associations without evidence for substantial heterogeneity or bias, but they might be genuine as they are the strongest associations reported in the literature in terms of magnitude of risk. The risk estimates are about twofold for individuals with diabetes. ${ }^{41}{ }^{46}$ Moreover, our evaluation indicated that the association between type 2 diabetes and intrahepatic cholangiocarcinoma was the most robust. Hepatocellular carcinomas and intrahepatic cholangiocarcinomas could have structural similarities, and they have been shown to share some common risk factors. ${ }^{54}{ }^{55}$ The meta-analyses of type 2 diabetes and risk of developing hepatocellular and pancreatic cancer, however, had $\mathrm{I}^{2}$ values approaching $100 \%$, and this substantial heterogeneity was not explained when alcohol consumption, history of cirrhosis, hepatitis B or C virus infection, obesity, and smoking status were taken into account. ${ }^{56}$

We did not identify prominent bias in the literature of type 2 diabetes and risk of developing cancer in terms of either the excess significance test or the small study effects test, with relatively few exceptions. There could be other types of biases or confounding, and the statistical tests we used are not designed to investigate these. There are many modifiable risk factors shared between type 2 diabetes and cancer, including age, sex, 
obesity, physical activity, diet, alcohol, and smoking, and some residual confounding is definitively possible as shown in our evaluation in which about $30 \%$ of the included studies adjusted only for age and sex. Reverse causality is another potential alternative hypothesis for the associations observed, specifically for those between type 2 diabetes and risk of developing hepatocellular and pancreatic cancer because it has been shown that these cancers can lead to dysfunction of insulin secretion or general derangement of glycaemic control. ${ }^{3}$ Evidence suggests, however, that it is unlikely for reverse causation to be entirely responsible for all the observed associations. ${ }^{356}$ Ascertainment bias might also exist as an increased probability of detection of cancer in the patients with diabetes, particularly in the early stages after onset, as there is heightened medical investigation of patients with a new diagnosis of diabetes, but studies have still found significant associations even after the exclusion of the first few years of follow-up after diagnosis. ${ }^{3}{ }^{37}$

Fewer studies exist for the association between type 2 diabetes and risk of death from cancer. Summary estimates in the meta-analyses were generally consistent with the results noted for incidence of cancer, but none of the associations for mortality passed our assessment for robust associations without evidence of bias because many of these associations did not reach significance at $\mathrm{P} \leq 0.001$. A recent pooled analysis of 97 prospective studies from the Emerging Risk Factors Collaboration (ERFC) reported a hazard ratio of 1.25 (95\% confidence interval 1.19 to 1.31 ) for total cancer death in people diabetes compared with those without diabetes; and diabetes was also moderately but significantly associated with death from cancers of the liver, pancreas, colorectum, and lung at $\mathrm{P}<0.001$ and also bladder, breast, and ovary at $\mathrm{P} \leq 0.05-0.001 .^{58}$ The list of these cancer sites overlaps partially with the sites for which we also documented the most robust (for breast and colorectal cancer; endometrial cancer and cholangiocarcinomas were not assessed separately by the ERFC) or strongest associations in magnitude for cancer incidence (liver and pancreas). We did not include this pooled analysis in our evaluation because study specific results were not available for evaluation. Of note, even in the exemplary ERFC analysis, data from cancer specific deaths were included only from subsets of the 97 cohorts (ranging from 20/97 for ovarian cancer to 60/97 for colorectal cancer), highlighting the problem of small numbers and missing data for cause specific deaths. For some cancer sites, most of the cancer deaths in ERFC had actually been recorded in just two cohorts, the Nurses Health Study and the Health Professionals Follow-up Study. Moreover, analyses of type 2 diabetes and mortality from cancer usually face more methodological concerns than studies for incidence of cancer. ${ }^{57} 59$ Cancer mortality studies have usually not taken into account stage at cancer diagnosis, and it has been shown that diabetes is associated with more advanced stage disease, particularly for breast cancer. ${ }^{59}$ These studies also do not take cancer treatments into account, and there is considerable evidence that oncologists might modify anticancer treatments in patients with cancer and diabetes because of increased rates of adverse effects and complications. ${ }^{59}$ We did not include meta-analyses for the association between type 2 diabetes and outcomes after cancer diagnosis among patients with cancer in this umbrella review because few such meta-analyses exist ${ }^{60-63}$ and the complexities reported above become even more prominent in this type of research.

\section{Strengths, weaknesses, and future research}

Most epidemiological studies of type 2 diabetes and developing of cancer or death from cancer have used self reported assessments of prevalent diabetes. Self reported measures of diabetes status have been previously shown to be over $99 \%$ specific and $66 \%$ sensitive compared with medical records. ${ }^{64}$ In addition, misclassification of patients with diabetes who did not know that they had the disease to the referent group of participants without diabetes is highly likely given that $46 \%$ of the estimated prevalence of diabetes is in people with undiagnosed disease. ${ }^{1}$ Most misclassification would probably have been non-differential as the assessment of diabetes was performed at baseline, long before cancer diagnosis in most cohorts, but it might be differential in case-control studies. Future prospective studies on type 2 diabetes and cancer incidence or mortality should incorporate incident cases of diabetes and use time varying exposure (diabetes duration, control (glycosylated haemoglobin), treatment, and complications) and confounder information to better model the association between diabetes and cancer. In addition, in the absence of data from randomised controlled trials for diabetes and risk of developing cancer, Mendelian randomisation analyses might prove useful in determining whether an observed association is likely to be causal. There is still a paucity of data and large heterogeneity in the associations for less common malignancies, and large consortiums of prospective studies should be carried out to better characterise the associations between type 2 diabetes and risk of cancer diagnosis and death. ${ }^{65}$ Several additional limitations and caveats should be considered in the interpretation of our findings. Firstly, we included only studies used in certain published meta-analyses and thus might have missed some individual studies if those were not identified in the original systematic searches. We think this unlikely as the summary results of the included meta-analyses were similar to the results of other duplicate but independent meta-analyses efforts on the same cancer outcomes. Secondly, both asymmetry and excess significance tests offer hints of bias, not definitive proof thereof, but our estimates are likely to be conservative as a negative test result does not exclude the potential for bias. Finally, it was beyond the scope of this review to explore the relation between type 1 diabetes or antidiabetic drugs and cancer risk.

\section{Conclusions}

In conclusion, although type 2 diabetes has been extensively studied in relation to risk of developing cancer and death from cancer and strong claims of significance exist for most of the studied associations, only a minority of these associations have robust support without hints of bias. Other associations could be genuine, but there is still substantial uncertainty about them. Future prospective studies and large consortiums with better assessment of type 2 diabetes and its time varying treatment, control, and sequelae and comprehensive standardised reporting of analyses are needed to draw firmer conclusions.

Contributors: All authors were involved in formulating the hypothesis and the design of the study protocol. The study hypothesis arose before inspection of the data. All authors had full access to all of the data in the study and can take responsibility for the integrity of the data and the accuracy of the data analysis. JCK and KKT carried out the search of the literature, data abstraction, data management, and statistical analysis. EEN and DSL verified several inputs of the final database of meta-analyses. KKT, JCK, and JPAI wrote the first draft; and all authors contributed to further drafts and approved the final version submitted. KKT and JCK contributed equally to this work. KKT and JPAI are guarantors.

Funding: This work was supported by the seventh framework programme of the European Union (PIEF-GA-2010-276017 to KKT and JPAI). The 


\section{What is already known on this topic}

Multiple studies and meta-analyses have claimed that type 2 diabetes is associated with an increased risk of developing cancer at several sites including liver, pancreas, endometrium, colorectum, breast, and bladder, and with a decreased risk of prostate cancer If causal, these associations would be of great importance for public health given the substantial global burden of these diseases Some claimed associations might be caused by biases in the literature, in particular selective reporting biases favouring the publication of significant associations, and causing either false positive or inflated estimates of association

\section{What this study adds}

Only a minority of associations between type 2 diabetes and risk of developing cancer or death from cancer for 20 cancer sites have robust supporting evidence without hints of bias

Evidence could be substantiated only for the associations between type 2 diabetes and risk of developing breast, intrahepatic cholangiocarcinoma, colorectal, and endometrial cancer

funding source detailed above had no role in the design, conduct, analysis, or reporting of the study. All authors carried out this research independently of the funding body.

Competing interests: All authors have completed the ICMJE uniform disclosure form at www.icmje.org/coi_disclosure.pdf and declare: no support from any organisation for the submitted work; no financial relationships with any organisations that might have an interest in the submitted work in the previous three years; no other relationships or activities that could appear to have influenced the submitted work.

\section{Ethical approval: Not required.}

Transparency declaration: The lead authors (the manuscript's guarantors) affirm that the manuscript is an honest, accurate, and transparent account of the study being reported; that no important aspects of the study have been omitted; and that any discrepancies from the study as planned have been explained.

Data sharing: No additional data available.

1 International Diabetes Federation. Diabetes atlas. International Diabetes Federation, 2013.

2 World Health Organization. The global burden of disease: 2004 update. WHO, 2008.

3 Giovannucci E, Harlan DM, Archer MC, Bergenstal RM, Gapstur SM, Habel LA, et al. Diabetes and cancer: a consensus report. Diabetes Care 2010;33:1674-85.

4 Dwan K, Altman DG, Arnaiz JA, Bloom J, Chan AW, Cronin E, et al. Systematic review of the empirical evidence of study publication bias and outcome reporting bias. PLoS One 2008;3:e3081.

5 loannidis JP. Why most published research findings are false. PLoS Med 2005;2:e124.

6 loannidis JP. Why most discovered true associations are inflated. Epidemiology 2008;19:640-8.

7 loannidis JP, Zhou Y, Chang CQ, Schully SD, Khoury MJ, Freedman AN. Potential increased risk of cancer from commonly used medications: an umbrella review of meta-analyses. Ann Oncol 2014;25:16-23.

8 Schoenfeld JD, loannidis JP. Is everything we eat associated with cancer? A systematic cookbook review. Am J Clin Nutr 2013;97:127-34.

9 Tsilidis KK, Papatheodorou SI, Evangelou E, Ioannidis JP. Evaluation of excess statistical significance in meta-analyses of 98 biomarker associations with cancer risk. J Natl Cancer Inst 2012;104:1867-78.

10 Boffetta P, McLaughlin JK, La Vecchia C, Tarone RE, Lipworth L, Blot WJ. False-positive results in cancer epidemiology: a plea for epistemological modesty. J Natl Cancer Inst 2008; 100:988-95.

11 Kyzas PA, Denaxa-Kyza D, loannidis JP. Almost all articles on cancer prognostic markers report statistically significant results. Eur J Cancer 2007;43:2559-79.

12 Kyzas PA, Loizou KT, loannidis JP. Selective reporting biases in cancer prognostic factor studies. J Natl Cancer Inst 2005;97:1043-55.

13 McLaughlin JK, Tarone RE. False positives in cancer epidemiology. Cancer Epidemiol Biomarkers Prev 2013;22:11-5.

14 DerSimonian R, Laird N. Meta-analysis in clinical trials. Control Clin Trials 1986;7:177-88.

15 Riley RD, Higgins JP, Deeks JJ. Interpretation of random effects meta-analyses. BMJ 2011;342:d549.

16 Higgins JP, Thompson SG. Quantifying heterogeneity in a meta-analysis. Stat Med 2002;21:1539-58.

17 loannidis JP, Patsopoulos NA, Evangelou E. Uncertainty in heterogeneity estimates in meta-analyses. BMJ 2007;335:914-6.

18 Sterne JA, Sutton AJ, loannidis JP, Terrin N, Jones DR, Lau J, et al. Recommendations for examining and interpreting funnel plot asymmetry in meta-analyses of randomised controlled trials. BMJ 2011;343:d4002.

19 Egger M, Davey Smith G, Schneider M, Minder C. Bias in meta-analysis detected by a simple, graphical test. BMJ 1997;315:629-34

20 Harbord RM, Egger M, Sterne JA. A modified test for small-study effects in meta-analyses of controlled trials with binary endpoints. Stat Med 2006;25:3443-57.

21 Peters JL, Sutton AJ, Jones DR, Abrams KR, Rushton L. Comparison of two methods to detect publication bias in meta-analysis. JAMA 2006;295:676-80.

22 Moreno SG, Sutton AJ, Ades AE, Stanley TD, Abrams KR, Peters JL, et al. Assessment of regression-based methods to adjust for publication bias through a comprehensive simulation study. BMC Med Res Methodol 2009;9:2.

23 Rucker G, Schwarzer G, Carpenter JR, Binder H, Schumacher M. Treatment-effect estimates adjusted for small-study effects via a limit meta-analysis. Biostatistics 2011;12:122-42.
24 Ioannidis JP, Trikalinos TA. An exploratory test for an excess of significant findings. Clin Trials 2007;4:245-53.

25 Ioannidis J. Clarifications on the application and interpretation of the test for excess significance and its extensions. J Math Psychol 2013;57:184-7.

26 Tsilidis KK, Panagiotou OA, Sena ES, Aretouli E, Evangelou E, Howells DW, et al. Evaluation of excess significance bias in animal studies of neurological diseases. PLOS Biol 2013;11:e1001609.

27 Hsieh FY, Bloch DA, Larsen MD. A simple method of sample size calculation for linear and logistic regression. Stat Med 1998;17:1623-34.

28 Schoenfeld DA. Sample-size formula for the proportional-hazards regression model. Biometrics 1983;39:499-503.

29 Ioannidis JP, Tarone R, McLaughlin JK. The false-positive to false-negative ratio in epidemiologic studies. Epidemiology 2011;22:450-6.

30 Sterne JA, Davey Smith G. Sifting the evidence-what's wrong with significance tests? BMJ 2001;322:226-31.

31 Johnson VE. Revised standards for statistical evidence. Proc Natl Acad Sci U S A 2013;110:19313-7.

32 Zhu Z, Wang X, Shen Z, Lu Y, Zhong S, Xu C. Risk of bladder cancer in patients with diabetes mellitus: an updated meta-analysis of 36 observational studies. BMC Cancer 2013;13:310.

33 Larsson SC, Mantzoros CS, Wolk A. Diabetes mellitus and risk of breast cancer: a meta-analysis. Int $J$ Cancer 2007;121:856-62.

34 Jing W, Jin G, Zhou X, Zhou Y, Zhang Y, Shao C, et al. Diabetes mellitus and increased risk of cholangiocarcinoma: a meta-analysis. Eur J Cancer Prev 2012;21:24-31.

35 Jiang Y, Ben Q, Shen H, Lu W, Zhang Y, Zhu J. Diabetes mellitus and incidence and mortality of colorectal cancer: a systematic review and meta-analysis of cohort studies. Eur J Epidemiol 2011;26:863-76

36 Friberg E, Orsini N, Mantzoros CS, Wolk A. Diabetes mellitus and risk of endometrial cancer: a meta-analysis. Diabetologia 2007;50:1365-74.

37 Huang W, Ren H, Ben Q, Cai Q, Zhu W, Li Z. Risk of esophageal cancer in diabetes mellitus: a meta-analysis of observational studies. Cancer Causes Control 2012;23:263-72.

38 Ren HB, Yu T, Liu C, Li YQ. Diabetes mellitus and increased risk of biliary tract cancer: systematic review and meta-analysis. Cancer Causes Control 2011;22:837-47.

39 Ge Z, Ben Q, Qian J, Wang Y, Li Y. Diabetes mellitus and risk of gastric cancer: a systematic review and meta-analysis of observational studies. Eur $J$ Gastroenterol Hepatol 2011:23:1127-35.

40 Tian T, Zhang LQ, Ma XH, Zhou JN, Shen J. Diabetes mellitus and incidence and mortality of gastric cancer: a meta-analysis. Exp Clin Endocrinol Diabetes 2012;120:217-23.

41 Wang $P$, Kang D, Cao W, Wang Y, Liu Z. Diabetes mellitus and risk of hepatocellular carcinoma: a systematic review and meta-analysis. Diabetes Metab Res Rev 2012;28:109-22.

42 Bao C, Yang X, Xu W, Luo H, Xu Z, Su C, et al. Diabetes mellitus and incidence and mortality of kidney cancer: a meta-analysis. J Diabetes Complications 2013;27:357-64.

43 Castillo JJ, Mull N, Reagan JL, Nemr S, Mitri J. Increased incidence of non-Hodgkin lymphoma, leukemia, and myeloma in patients with diabetes mellitus type 2: a meta-analysis of observational studies. Blood 2012;119:4845-50.

44 Lee JY, Jeon I, Lee JM, Yoon JM, Park SM. Diabetes mellitus as an independent risk factor for lung cancer: a meta-analysis of observational studies. Eur $\mathrm{J}$ Cancer 2013;49:2411-23.

45 Lee JY, Jeon I, Kim JW, Song YS, Yoon JM, Park SM. Diabetes mellitus and ovarian cancer risk: a systematic review and meta-analysis of observational studies. Int $J$ Gynecol Cancer 2013;23:402-12.

46 Ben Q, Xu M, Ning X, Liu J, Hong S, Huang W, et al. Diabetes mellitus and risk of pancreatic cancer: A meta-analysis of cohort studies. Eur J Cancer 2011;47:1928-37.

47 Bansal D, Bhansali A, Kapil G, Undela K, Tiwari P. Type 2 diabetes and risk of prostate cancer: a meta-analysis of observational studies. Prostate Cancer Prostatic Dis 2013;16:151-8,S1.

48 Schmid D, Behrens G, Jochem C, Keimling M, Leitzmann M. Physical activity, diabetes, and risk of thyroid cancer: a systematic review and meta-analysis. Eur J Epidemiol 2013;28:945-58

49 Noto H, Tsujimoto T, Sasazuki T, Noda M. Significantly increased risk of cancer in patients with diabetes mellitus: a systematic review and meta-analysis. Endocr Pract 2011;17:616-28.

50 Kavvoura FK, Liberopoulos G, loannidis JP. Selection in reported epidemiological risks: an empirical assessment. PLoS Med 2007;4:e79.

51 loannidis JP. The importance of potential studies that have not existed and registration of observational data sets. JAMA 2012;308:575-6.

52 Nickerson R. Confirmation bias: a ubiquitous phenomenon in many guises. Rev Gen Psychol 1998;2:175-220

53 Klayman J, Y-W H. Confirmation, disconfirmation, and information in hypothesis testing. Psychol Rev 1987;94:211-28.

54 Shaib YH, El-Serag HB, Davila JA, Morgan R, McGlynn KA. Risk factors of intrahepatic cholangiocarcinoma in the United States: a case-control study. Gastroenterology 2005;128:620-6 
55 Lee TY, Lee SS, Jung SW, Jeon SH, Yun SC, Oh HC, et al. Hepatitis B virus infection and intrahepatic cholangiocarcinoma in Korea: a case-control study. Am J Gastroenterol 2008;103:1716-20

56 Shikata K, Ninomiya T, Kiyohara Y. Diabetes mellitus and cancer risk: review of the epidemiological evidence. Cancer Sci 2013;104:9-14.

57 Johnson JA, Carstensen B, Witte D, Bowker SL, Lipscombe L, Renehan AG. Diabetes and cancer (1): evaluating the temporal relationship between type 2 diabetes and cancer incidence. Diabetologia 2012;55:1607-18.

58 Seshasai SR, Kaptoge S, Thompson A, Di Angelantonio E, Gao P, Sarwar N, et al Diabetes mellitus, fasting glucose, and risk of cause-specific death. $N$ Engl J Med 2011;364:829-41.

59 Renehan AG, Yeh HC, Johnson JA, Wild SH, Gale EA, Moller H. Diabetes and cancer (2): evaluating the impact of diabetes on mortality in patients with cancer. Diabetologia 2012;55:1619-32.

60 Peairs KS, Barone BB, Snyder CF, Yeh HC, Stein KB, Derr RL, et al. Diabetes mellitus and breast cancer outcomes: a systematic review and meta-analysis. J Clin Oncol 2011;29:40-6.

61 Snyder CF, Stein KB, Barone BB, Peairs KS, Yeh HC, Derr RL, et al. Does pre-existing diabetes affect prostate cancer prognosis? A systematic review. Prostate Cancer Prostatic Dis 2010;13:58-64.

62 Stein KB, Snyder CF, Barone BB, Yeh HC, Peairs KS, Derr RL, et al. Colorectal cancer outcomes, recurrence, and complications in persons with and without diabetes mellitus: a systematic review and meta-analysis. Dig Dis Sci 2010;55:1839-51.
63 Barone BB, Yeh HC, Snyder CF, Peairs KS, Stein KB, Derr RL, et al. Long-term all-cause mortality in cancer patients with preexisting diabetes mellitus: a systematic review and meta-analysis. JAMA 2008;300:2754-64

64 Okura Y, Urban LH, Mahoney DW, Jacobsen SJ, Rodeheffer RJ. Agreement between self-report questionnaires and medical record data was substantial for diabetes, hypertension, myocardial infarction and stroke but not for heart failure. J Clin Epidemiol 2004:57:1096-103

65 Khoury MJ, Lam TK, loannidis JP, Hartge P, Spitz MR, Buring JE, et al. Transforming epidemiology for 21 st century medicine and public health. Cancer Epidemiol Biomarkers Prev 2013;22:508-16.

\section{Accepted: 27 October 2014}

\section{Cite this as: BMJ 2015;350:g7607}

This is an Open Access article distributed in accordance with the Creative Commons Attribution Non Commercial (CC BY-NC 4.0) license, which permits others to distribute, remix, adapt, build upon this work non-commercially, and license their derivative works on different terms, provided the original work is properly cited and the use is non-commercial. See: http://creativecommons.org/licenses/by-nc/4.0/. 


\section{Tables}

\begin{tabular}{|c|c|c|c|c|c|c|c|c|}
\hline \multirow[b]{2}{*}{ Study } & \multirow{2}{*}{$\begin{array}{c}\text { Association } \\
\text { between diabetes } \\
\text { and }^{*}\end{array}$} & \multirow[b]{2}{*}{$\begin{array}{c}\text { No of } \\
\text { cases/population }\end{array}$} & \multicolumn{3}{|c|}{ Summary relative risk $(95 \% \mathrm{Cl})$} & \multirow[b]{2}{*}{$\begin{array}{l}\text { Fixed } P \\
\text { value }\end{array}$} & \multirow[b]{2}{*}{$\begin{array}{l}\text { Random } \\
\mathrm{P} \text { value§ }\end{array}$} & \multirow{2}{*}{$\begin{array}{c}95 \% \\
\text { prediction } \\
\text { interval }\end{array}$} \\
\hline & & & Fixed effects & Random effects & Largest study† & & & \\
\hline Zhu, $2013^{32}$ & Bladder cancer & $50676 / 12500000$ & 1.26 (1.22 to 1.29$)$ & $1.35(1.17$ to 1.56$)$ & $0.96(0.92$ to 1.01$)$ & $<0.001$ & $<0.001$ & 0.61 to 3.02 \\
\hline Larsson, $2007^{33}$ & Breast cancer & 30 859/1 422788 & 1.19 (1.16 to 1.23$)$ & $1.20(1.12$ to 1.28$)$ & $1.20(1.10$ to 1.20$)$ & $<0.001$ & $<0.001$ & 1.01 to 1.43 \\
\hline Larsson, $2007^{33}$ & $\begin{array}{l}\text { Breast cancer } \\
\text { mortality }\end{array}$ & $4442 / 1090597$ & $1.21(1.10$ to 1.34$)$ & 1.24 (0.95 to 1.62$)$ & $1.27(1.11$ to 1.45$)$ & $<0.001$ & 0.11 & 0.49 to 3.16 \\
\hline Jing, $2012^{34}$ & ICC & $3152 / 1041385$ & 1.98 (1.77 to 2.21$)$ & $1.97(1.57$ to 2.46$)$ & $1.80(1.50$ to 2.10$)$ & $<0.001$ & $<0.001$ & 1.11 to 3.49 \\
\hline Jing, $2012^{34}$ & ECC & $2431 / 1995190$ & $1.63(1.46$ to 1.81$)$ & $1.63(1.29$ to 2.05$)$ & $1.50(1.30$ to 1.80$)$ & $<0.001$ & $<0.001$ & 0.86 to 3.08 \\
\hline Jiang, $2011^{35}$ & Colorectal cancer & $61690 / 8244732$ & 1.25 (1.22 to 1.29$)$ & $1.27(1.21$ to 1.34$)$ & $1.41(1.32$ to 1.50$)$ & $<0.001$ & $<0.001$ & 1.07 to 1.52 \\
\hline Jiang, $2011^{35}$ & $\begin{array}{l}\text { Colorectal cancer } \\
\text { mortality }\end{array}$ & $4394 / 4090537$ & $1.34(1.27$ to 1.40$)$ & 1.20 (1.03 to 1.40$)$ & $1.54(1.44$ to 1.65$)$ & $<0.001$ & 0.02 & 0.74 to 1.94 \\
\hline Friberg, $2007^{36}$ & Endometrial cancer & $8174 / 242881$ & 1.78 (1.67 to 1.91$)$ & $1.97(1.71$ to 2.27$)$ & $1.80(1.60$ to 2.00$)$ & $<0.001$ & $<0.001$ & 1.23 to 3.16 \\
\hline Friberg, $2007^{36}$ & $\begin{array}{l}\text { Endometrial cancer } \\
\text { mortality }\end{array}$ & $103 / 671964$ & 1.14 (0.89 to 1.46$)$ & $1.23(0.78$ to 1.93$)$ & 1.33 (0.92 to 1.90$)$ & 0.29 & 0.38 & 0.28 to 5.36 \\
\hline Huang, $2011^{37}$ & $\begin{array}{l}\text { Oesophageal } \\
\text { cancer }\end{array}$ & $3001 / 2486812$ & 1.30 (1.18 to 1.42$)$ & $1.30(1.12$ to 1.50$)$ & 1.66 (1.35 to 2.05$)$ & $<0.001$ & $<0.001$ & 0.86 to 1.95 \\
\hline Ren, $2011^{38}$ & Gallbladder cancer & $1821 / 2563936$ & 1.44 (1.28 to 1.62$)$ & 1.52 (1.26 to 1.84$)$ & $1.30(1.10$ to 1.50$)$ & $<0.001$ & $<0.001$ & 0.99 to 2.33 \\
\hline Ge, $2011^{39}$ & Gastric cancer & 15 970/8 097417 & 1.08 (1.05 to 1.12$)$ & 1.09 (0.98 to 1.22$)$ & $1.12(1.05$ to 1.19$)$ & $<0.001$ & 0.12 & 0.72 to 1.65 \\
\hline Tian, $2012^{40}$ & $\begin{array}{l}\text { Gastric cancer } \\
\text { mortality }\end{array}$ & $2447 / 2556574$ & 1.24 (1.15 to 1.33 ) & 1.29 (1.04 to 1.59$)$ & 1.15 (1.04 to 1.26$)$ & $<0.001$ & 0.02 & 0.66 to 2.49 \\
\hline Wang, $2012^{41}$ & $\mathrm{HCC}$ & 33 765/7 210641 & 1.73 (1.68 to 1.77$)$ & 2.31 (1.87 to 2.84$)$ & 1.21 (1.17 to 1.25$)$ & $<0.001$ & $<0.001$ & 0.66 to 8.02 \\
\hline Wang, $2012^{41}$ & HCC mortality & 292/1 156796 & 2.22 (1.95 to 2.53 ) & 2.43 (1.67 to 3.55$)$ & 2.48 (2.07 to 2.97$)$ & $<0.001$ & $<0.001$ & 0.78 to 7.54 \\
\hline Bao, $2013^{42}$ & Kidney cancer & 12 980/6 529381 & 1.38 (1.32 to 1.44$)$ & 1.38 (1.10 to 1.72$)$ & 1.09 (1.03 to 1.16$)$ & $<0.001$ & 0.006 & 0.55 to 3.44 \\
\hline Bao, $2013^{42}$ & $\begin{array}{l}\text { Kidney cancer } \\
\text { mortality }\end{array}$ & $2646 / 2347294$ & 1.16 (1.01 to 1.33$)$ & 1.16 (1.01 to 1.33 ) & 1.25 (1.03 to 1.53$)$ & 0.04 & 0.04 & 0.97 to 1.37 \\
\hline Lee, $2013^{44}$ & Lung cancer & $207454 / 15900000$ & 0.88 (0.87 to 0.90$)$ & $1.03(0.94$ to 1.13$)$ & $0.79(0.77$ to 0.80$)$ & $<0.001$ & 0.49 & 0.70 to 1.52 \\
\hline Castillo, $2012^{43}$ & Leukaemia & $4156 / 6447905$ & 1.28 (1.22 to 1.34$)$ & 1.28 (1.05 to 1.57$)$ & $1.18(1.11$ to 1.25$)$ & $<0.001$ & 0.02 & 0.66 to 2.48 \\
\hline Castillo, $2012^{43}$ & Multiple myeloma & $3051 / 5673856$ & 1.10 (1.03 to 1.17$)$ & 1.27 (0.98 to 1.64$)$ & 0.98 (0.91 to 1.06$)$ & 0.003 & 0.07 & 0.56 to 2.86 \\
\hline Castillo, $2012^{43}$ & $\begin{array}{l}\text { Non-Hodgkin's } \\
\text { lymphoma }\end{array}$ & 12 353/6 070099 & 1.10 (1.06 to 1.15$)$ & 1.27 (1.09 to 1.48$)$ & 0.96 (0.91 to 1.01$)$ & $<0.00$ & 0.002 & 0.70 to 2.30 \\
\hline Lee, $2013^{45}$ & Ovarian cancer & $7651 / 2173612$ & 1.12 (1.03 to 1.22$)$ & $1.17(1.02$ to 1.34$)$ & 1.05 (0.93 to 1.20$)$ & 0.008 & 0.02 & 0.79 to 1.72 \\
\hline Ben, $2011^{46}$ & Pancreatic cancer & $52445 / 10006592$ & 2.22 (2.15 to 2.29 ) & 1.95 (1.66 to 2.28$)$ & 3.22 (3.03 to 3.42 ) & $<0.001$ & $<0.001$ & 0.87 to 4.34 \\
\hline Bansal, $2013^{47}$ & Prostate cancer & 135 970/8 197926 & 0.89 (0.88 to 0.91$)$ & 0.91 (0.82 to 1.01$)$ & 0.89 (0.87 to 0.91$)$ & $<0.001$ & 0.07 & 0.49 to 1.69 \\
\hline Schmid, $2013^{48}$ & Thyroid cancer & $1230 / 960840$ & 1.16 (0.97 to 1.39$)$ & 1.16 (0.97 to 1.39$)$ & 1.19 (0.90 to 1.57$)$ & 0.09 & 0.09 & 0.93 to 1.45 \\
\hline Noto, $2011^{49}$ & Total cancer & 38 010/1 859420 & 1.11 (1.09 to 1.13$)$ & $1.10(1.04$ to 1.17$)$ & $1.10(1.07$ to 1.13$)$ & $<0.001$ & 0.002 & 0.90 to 1.35 \\
\hline Noto, $2011^{49}$ & $\begin{array}{l}\text { Total cancer } \\
\text { mortality }\end{array}$ & 11 386/2 320578 & 1.21 (1.17 to 1.25$)$ & 1.16 (1.04 to 1.30$)$ & 1.28 (1.23 to 1.33$)$ & $<0.001$ & 0.008 & 0.80 to 1.70 \\
\hline
\end{tabular}

$\mathrm{HCC}=$ hepatocellular carcinoma; ICC=intrahepatic cholangiocarcinoma; ECC=extrahepatic cholangiocarcinoma.

*Incidence unless otherwise specified.

†Relative risk and 95\% confidence interval of largest study (smallest SE) in each meta-analysis.

$\ddagger P$ value of summary fixed effects estimate.

$\S \mathrm{P}$ value of summary random effects estimate. 


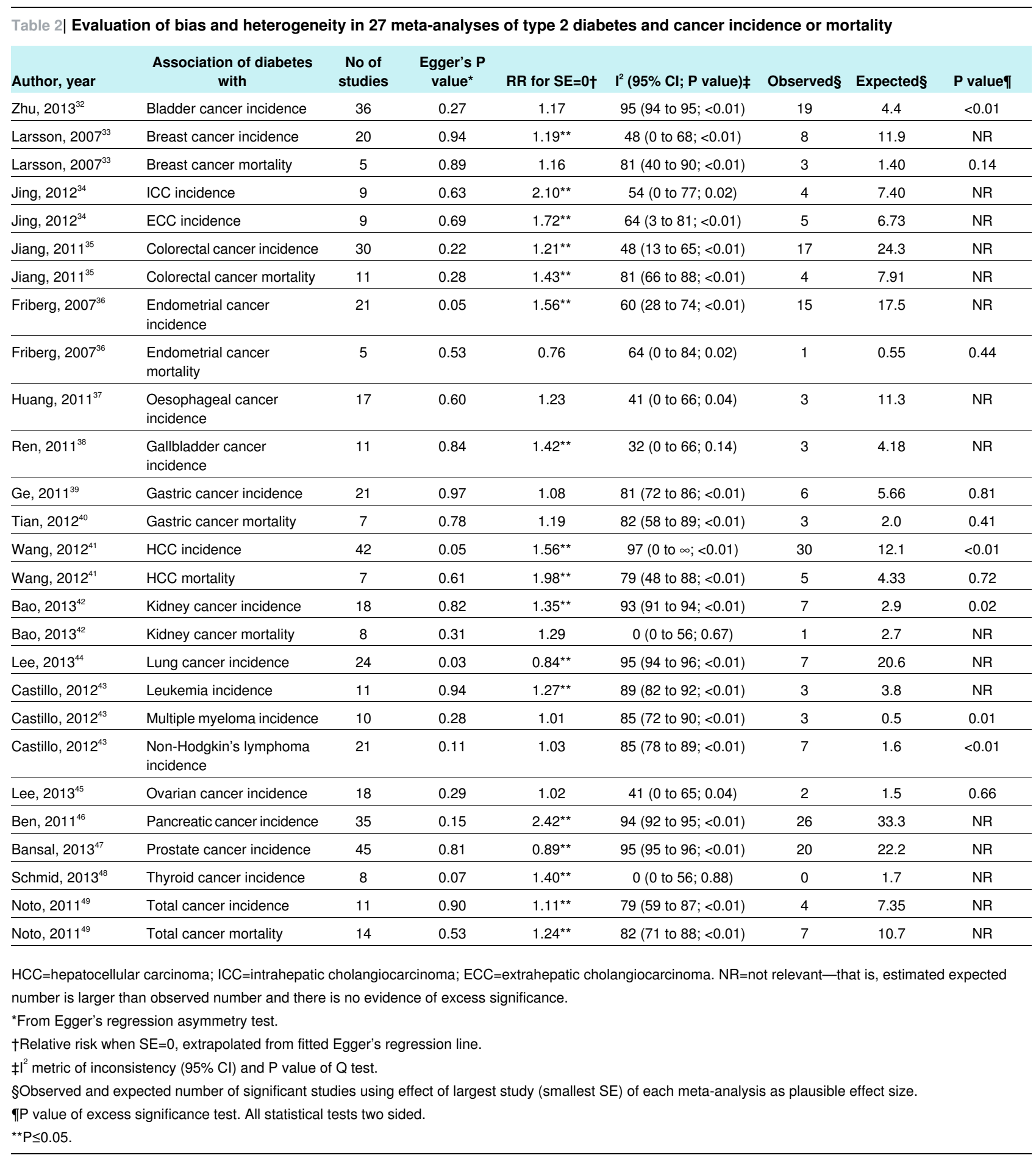




\section{Figures}

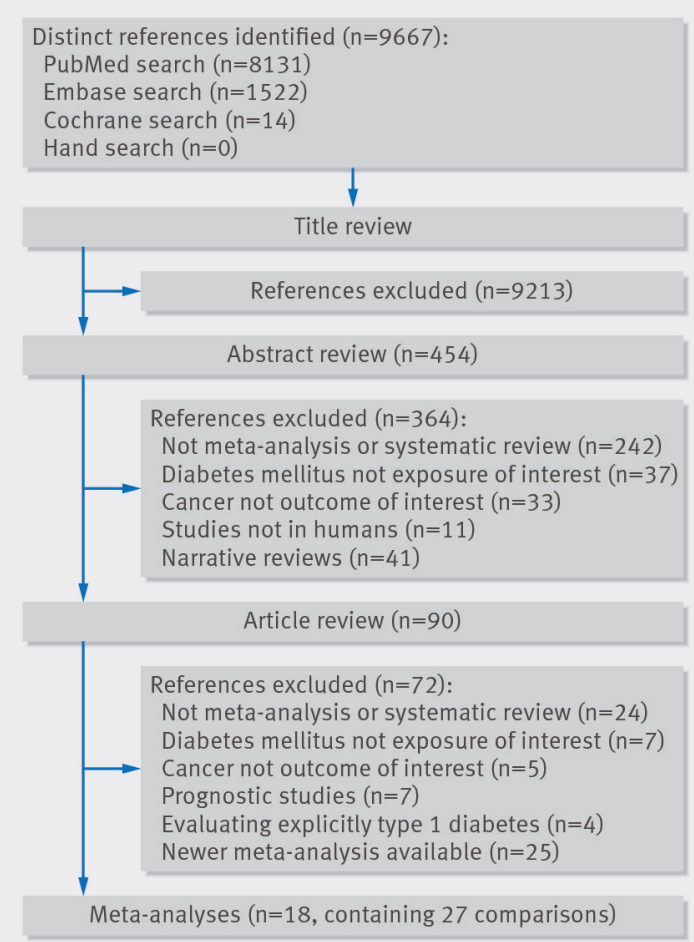

Fig 1 Flow diagram of selection process of meta-analyses on type 2 diabetes and cancer 


\begin{tabular}{|c|c|c|c|c|c|c|}
\hline Association of diabetes with & $\begin{array}{l}\text { No of } \\
\text { cases }\end{array}$ & & $\begin{array}{l}\text { Evidence } \\
\text { for bias }\end{array}$ & $\begin{array}{l}\text { Random effects }(95 \% \mathrm{Cl}) \\
\text { and } 95 \% \text { prediction intervals }\end{array}$ & $\begin{array}{c}\text { Random } \\
\text { effects }(95 \% \mathrm{Cl})\end{array}$ & $\begin{array}{l}95 \% \\
\text { prediction } \\
\text { intervals }\end{array}$ \\
\hline Prostate cancer incidence & 135970 & 95 & No & . & 0.91 (0.82 to 1.01$)$ & 0.49 to 1.69 \\
\hline Lung cancer incidence & 207454 & 95 & No & 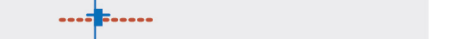 & $1.03(0.94$ to 1.13$)$ & $0.70 \circ 1.52$ \\
\hline Gastric cancer incidence & 15970 & 81 & Yes & 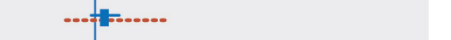 & 1.09 (0.98 to 1.22$)$ & 0.72 o 1.65 \\
\hline Total cancer incidence & 38010 & 79 & No & 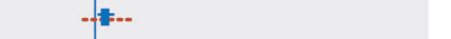 & $1.10(1.04$ to 1.17$)$ & 0.90 to 1.35 \\
\hline Kidney cancer mortality & 2646 & 0 & Yes & the. & $1.16(1.01$ to 1.33$)$ & 0.97 to 1.37 \\
\hline Thyroid cancer incidence & 1230 & 0 & No & $=$ & 1.16 (0.97 to 1.39$)$ & 0.93 to 1.45 \\
\hline Total cancer mortality & 11386 & 82 & No & $\ldots$ & $1.16(1.04$ to 1.30$)$ & 0.80 to 1.70 \\
\hline Ovarian cancer incidence & 7651 & 41 & Yes & |t=-.... & $1.17(1.02$ to 1.34$)$ & 0.79 to 1.72 \\
\hline Breast cancer incidence & 30859 & 48 & No & 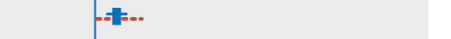 & $1.20(1.12$ to 1.28$)$ & 1.01 to 1.43 \\
\hline Colorectal cancer mortality & 4394 & 81 & No & - & $1.20(1.03$ to 1.40$)$ & 0.74 to 1.94 \\
\hline Endometrial cancer mortality & 103 & 64 & Yes & 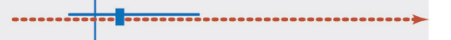 & $1.23(0.78$ to 1.93$)$ & 0.28 to 5.36 \\
\hline Breast cancer mortality & 4442 & 81 & Yes & -1F-.... & $1.24(0.95$ to 1.62$)$ & 0.49 to 3.16 \\
\hline Colorectal cancer incidence & 61690 & 48 & No & --*-- & $1.27(1.21$ to 1.34$)$ & 1.07 to 1.52 \\
\hline Multiple myeloma incidence & 3051 & 85 & Yes & - there.. & 1.27 (0.98 to 1.64$)$ & 0.56 to 2.86 \\
\hline Non-Hodgkin's lymphoma incidence & e 12353 & 85 & Yes & -DIf-........... & 1.27 (1.09 to 1.48$)$ & 0.70 to 2.30 \\
\hline Leukemia incidence & 4156 & 89 & No & -1]-............... & $1.28(1.05$ to 1.57$)$ & 0.66 to 2.48 \\
\hline Gastric cancer mortality & 2447 & 82 & Yes & 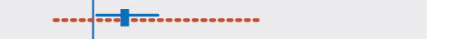 & $1.29(1.04$ to 1.59$)$ & 0.66 to 2.49 \\
\hline Esophageal cancer incidence & 3001 & 41 & Yes & 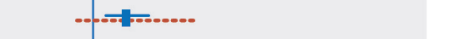 & $1.30(1.12$ to 1.50$)$ & 0.86 to 1.95 \\
\hline Bladder cancer incidence & 50676 & 95 & Yes & . तो-....... & 1.35 (1.17 to 1.56$)$ & 0.61 to 3.02 \\
\hline Kidney cancer incidence & 12980 & 93 & Yes & 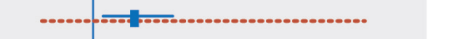 & 1.38 (1.10 to 1.72$)$ & 0.55 to 3.44 \\
\hline Gallbladder cancer incidence & 1821 & 32 & No & 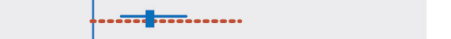 & $1.52(1.26$ to 1.84$)$ & 0.99 to 2.33 \\
\hline ECC incidence & 2431 & 64 & No & $\ldots$ & 1.63 (1.29 to 2.05$)$ & 0.86 to 3.08 \\
\hline Pancreatic cancer incidence & 52445 & 94 & No & ....... & 1.95 (1.66 to 2.28$)$ & 0.87 to 4.34 \\
\hline ICC incidence & 3152 & 54 & No & 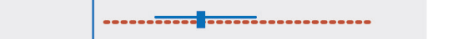 & 1.97 (1.57 to 2.46$)$ & 1.11 to 3.49 \\
\hline Endometrial cancer incidence & 8174 & 60 & No & 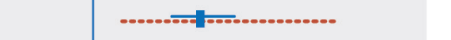 & 1.97 (1.71 to 2.27$)$ & 1.23 to 3.16 \\
\hline HCC incidence & 33765 & 97 & Yes & ......... & 2.31 (1.87 to 2.84$)$ & 0.66 to 8.02 \\
\hline \multirow[t]{2}{*}{ HCC mortality } & 292 & 79 & No & - & 2.43 (1.67 to 3.55$)$ & 0.78 to 7.54 \\
\hline & & & 0 & $\begin{array}{llllllll}0.5 & 1.0 & 1.5 & 2.0 & 2.5 & 3.0 & 3.5 & 4.0\end{array}$ & & \\
\hline
\end{tabular}

Fig 2 Summary random effects estimates with 95\% confidence and prediction intervals from 27 meta-analyses of type 2 diabetes and incidence of cancer or mortality (evidence for bias assessed with extrapolation of Egger regression line to study of infinite sample size and with excess significance test). HCC=hepatocellular carcinoma; ICC=intrahepatic cholangiocarcinoma; ECC=extrahepatic cholangiocarcinoma 